\title{
Importance of HCN channels involved in various body conditions
}

$<$ Luna Gonzalez, Alma Delia> (E-mail: almadelia097@gmail.com) a , <Galeana Rojas, Maria Fernanda $>$ (E-mail: ferga97rojas@hotmail.com) ${ }^{\mathrm{b}},<$ Jauregui Perez, Laura Jannete $>$ (E-mail: laurajannete@gmail.com) ${ }^{\mathrm{c}},<$ Paramo Ramirez, Arely $>$ (E-mail: arelyparamo@hotmail.com $)^{\mathrm{d}}$

$a<$ Mixtec Regional Campus headquarters Izúcar de Matamoros, Faculty of Biological Sciences, Benemérita Universidad Autónoma de Puebla, Puebla, Mexico >

$b<$ Department of Technologies Information, Universidad de la Salle Bajio, León, México.>

$c<$ Division of Natural and Exact Sciences, Guanajuato University, Guanajuato, México >

$d<$ Division of Natural and Exact Sciences, Guanajuato University, Guanajuato, México >

\begin{abstract}
.
HCN channels play a variety of important functions in our body, this can be from the control in the heart rate as in the central and peripheral nervous system. This type of channel is voltage dependent and has a permeability to $\mathrm{Na}^{+}$and $\mathrm{K}^{+}$, this permeability is usually controlled by the cAMP that is inside the cell. HCN channels participate in human diseases such as pain, heart failure, etc., and having a fundamental role in our body functions can offer new tools that allow the pharmaceutical industry to develop drugs, mainly analgesics.
\end{abstract}

Key words: HCN channels, pain, inhibitors, new drugs, epilepsy.

\section{Introduction}

HCN channels have a structure similar to voltage-dependent ion channels and can be heteromeric or homomeric [1]. Each subunit of the HCN channel has two main modules in its structure; the transmembrane domains and the cytosolic C-terminal, both works together for channel activation and function. Transmembrane domains contain six $\alpha$-helixes (S1-S6) and are usually associated in tetramers [2]. The C-terminal has a specific domain of binding to cyclic nucleotides. In mammals we can find four isoforms of this type of channel (HCN1- HCN4).

\section{Physical and Biological Characteristics of HCN Channels}

The HCN channels have a voltage sensor domain that has a charged S4 helix containing residues of the amino-acids arginine/lysine with a regulated space. HCN channels have a fundamental role in the signaling of sensory neurons, as well as play an excitability function, these functions are achieved by binding the C-terminal HCN channels to a domain of binding to intracellular cyclic nucleotides (CNBD). CNBD is coupled to the core of the channel by 80 amino-acid peptides called the C-linker, this facilitates the activation of the channel by changing the direction in the voltage on which the activation depends [1].

The HCN channels are distinguished by four unusual characteristics: the first is the activation of the channel carried out by hyperpolarization; the second is the ease of activation by the direct way of interacting with cAMP; then we have the great permeability of $\mathrm{Na}+$ and $\mathrm{K}+$; and the last is the inhibition by millimolar concentrations of $\mathrm{Cs}+[1]$. 
2. General Operation of HCN Channels

$\mathrm{HCN}$ channels are abundant in the brain and heart. HCN4 channels are activated by hyperpolarization after a cardiac action potential. Neurotransmitters or pharmacological intervention often raise cAMP levels in order to change the voltage dependence and then increase the conductivity of the current to the maximum of the HCN channels, this speeds up the heart rate. The HCN channels participate in different activities, such as the determination of the resting membrane potential, synaptic transmission, learning and integration between dentrites. HCN channels are also present in heart failure, pain and epilepsy [1].

\subsection{HCN Channel Relationship to Neurological Diseases}

$\mathrm{HCN}$ channels are widely distributed in the central and peripheral nervous system and play an important role in several basic functions, as well as being reported to be related to inflammatory and neuropathic pain. Recently, a genetic model in which selective deletion has been used has shown that HCN2 channels are present before and during pain. The results of this research suggest a strategy capable of developing new drug technologies to treat inflammatory pain.

Alteration of HCN channels in epilepsy was first demonstrated in rat febrile seizure models in 2001. Studies have shown that the mRNA of HCN2 had an increase in the hippocampus CA1, while the mRNA of HCN1 was reduced in the used model of febrile convulsions, after the results of these studies it has been proven that the HCN channels have an important role in epilepsy, since eliminating HCN1 obtained a greater susceptibility to convulsions and the elimination of HCN2 produced epilepsy of deep spontaneous absence, in both the same model of study was used.

\section{HCN Channel Inhibitor}

HCN channel inhibitors may decrease heart rate and aid in analgesic treatment, leading to the development of new drugs to help eradicate this problem. ivabradine is the first clinically used HCN channel inhibitor, its use was approved in 2005 by the European Medicines Agency for chronic stable angina pectoris.

$\mathrm{HCN}$ channel inhibitors are a group of compounds that contain bradines in their structure. Zatebradine, cilobradine and ivabradine make up this group. Ivabradine has been shown to have effects on heart rate slowing and inhibits HCN channels. ivabradine penetrates into the pore of the channel and competes with ions at the bonding sites, the more ions flow through the HCN channels, the more efficiently ivabradine acts at the bonding sites of the pores. Ivabradine has also been reported with benefits in patients with systolic and diastolic heart failure [1].

\section{Conclusions}

The HCN channels (voltage dependent channels and modulated by cyclic nucleotides) allow the access of $\mathrm{K}^{+}$and $\mathrm{Na}^{+}$ions. They are found more easily in different nerve regions, as well as in the heart and are related to diseases where pain persists. Several studies have shown that they contribute to physiological processes, such as learning and memory, but also in pathological processes, such as epilepsy or neuropathic pain. Studies conducted on the inhibitors of these channels result in an impressive finding in the creation of new drugs that can treat pain and other conditions in human physiology.

\section{References}

[1] CAO, Y., PANG, J. Y ZHOU, P.

HCN Channel as Therapeutic Targets for Heart Failure and Pain 
Bibliografía: 2. Cao Y, Pang J, Zhou P. HCN Channel as Therapeutic Targets for Heart Failure and Pain. Current Topics in Medicinal Chemistry. 2016;16(16):1855-1861.

\section{[2] TALEISNIK, S.}

Receptores celulares y la transducción de señales. Temas de Biología Celular

Bibliografía: 1. Taleisnik S. Receptores celulares y la transducción de señales. Temas de Biología Celular [Internet]. Google Books. 2006 [cited 27 June 2019]. Available from: https://books.google.es/books?id=wFWEimjnGr4C\&pg=PA186\&dq=canales + de + hcn\&hl=es419\&sa $=$ X\&ved=0ahUKEwiz17HFxo7jAhWZilwKHd1vB_cQ6AEIKTAA\#v=onepage $\& \mathrm{q}=$ canales $\%$ $20 \mathrm{de} \% 2$ 